\title{
Evolution of Vocal Communication in Birds: Environmental Adaptation and Sexual Selection
}

\author{
Kazuo OKANOYA \\ Faculty of Letters, Chiba University 1-33 Yayoi-cho, Inage-ku, 263-8522, Japan and PRESTO, \\ Japan Science and Technology Corporation, Kawaguchi, Saitama 332-0012, Japan
}

Diverse vocal signals have evolved as means of communication in several classes of animals. Bird vocalizations especially have attracted our attention because of their aesthetic compositions. Birds and us humans certainly share common sense as to the beauty of musical sounds. More recently, scientific investigations on bird vocalizations revealed their mechanisms, functions, and evolution.

The following three papers included in this volume are contributions from the speakers at the symposium entitled "Evolution of vocal communication in birds: environmental adaptation and sexual selection" held as a part of the 1999 annual meeting of the Ornithological Society of Japan.

In this symposium, we discussed specific and general questions in the evolution of avian vocal signals. Eugene Morton first introduced general features of avian vocal communication that were common to other classes of animals. He then explained specific features of avian communication system. Teruyo Oba next discussed factors that produced similarity and variation in avian vocal signals. Factors such as systematic, environmental, ecological, and social were discussed. Kazuo Okanoya focused on the issue of female choice and evolution of vocal complexity in Bengalese finches. Shoji Hamao explained the strategies of male birds.

Each of these papers is not a simple written version of the symposium talk, but rather an extension of it. Each of us prepared our paper so that we could echo the exciting discussion we had at the symposium. We are most grateful to the editors of Japanese Journal of Ornithology for their help and patience during the process of preparation for this volume. 